\title{
Niche differentiation of two sympatric species of Microdochium colonizing the roots of common reed
}

\author{
Michael Ernst ${ }^{1}$, Karin Neubert ${ }^{1}$, Kurt W Mendgen ${ }^{1}$ and Stefan GR Wirsel ${ }^{2,3^{*}}$
}

\begin{abstract}
Background: Fungal endophyte communities are often comprised of many species colonizing the same host. However, little is known about the causes of this diversity. On the one hand, the apparent coexistence of closely related species may be explained by the traditional niche differentiation hypothesis, which suggests that abiotic and/or biotic factors mediate partitioning. For endophytes, such factors are difficult to identify, and are therefore in most cases unknown. On the other hand, there is the neutral hypothesis, which suggests that stochastic factors may explain high species diversity. There is a need to investigate to what extent each of these hypotheses may apply to endophytes.

Results: The niche partitioning of two closely related fungal endophytes, Microdochium bolleyi and M. phragmitis, colonizing Phragmites australis, was investigated. The occurrences of each species were assessed using specific nested-PCR assays for 251 field samples of common reed from Lake Constance, Germany. These analyses revealed niche preferences for both fungi. From three niche factors assessed, i.e. host habitat, host organ and season, host habitat significantly differentiated the two species. M. bolleyi preferred dry habitats, whereas M. phragmitis prevailed in flooded habitats. In contrast, both species exhibited a significant preference for the same host organ, i.e. roots. Likewise the third factor, season, did not significantly distinguish the two species. Differences in carbon utilization and growth temperature could not conclusively explain the niches. The inclusion of three unrelated species of Ascomycota, which also colonize P. australis at the same locations, indicated spatio-temporal niche partitioning between all fungi. None of the species exhibited the same preferences for all three factors, i.e. host habitat, host organ, and time of the season.

Conclusions: The fungal species colonizing common reed investigated in this study seem to exploit niche differences leading to a separation in space and time, which may allow for their coexistence on the same host. A purely neutral model is unlikely to explain the coexistence of closely related endophytes on common reed.
\end{abstract}

\section{Background}

Traditionally, biodiversity has been explained by the niche partitioning hypothesis, which stresses that coexisting species are differentiated by niche dimensions. On the other hand, the neutral hypothesis proposes that species at the same trophic level colonizing the same space are functionally equivalent [1], because different species have the same likelihood of dispersal, death and

\footnotetext{
* Correspondence: stefan.wirsel@landw.uni-halle.de

${ }^{2}$ Institut für Agrar- und Ernährungswissenschaften, Naturwissenschaftliche Fakultät III, Martin-Luther-Universität Halle-Wittenberg, Betty-Heimann-Str. 3, D-06120 Halle (Saale), Germany

Full list of author information is available at the end of the article
}

birth. Assessment of plant communities has yielded controversial results, some seemed to support the neutral hypothesis [1-3], whereas others did not [4,5]. Attempts have been made to resolve the controversy between the traditional and the neutral hypotheses by integrating stochastic factors into niche-based models [6]. A metaanalysis comparing 158 community studies, covering a wide range of habitats, regions and organisms indicated that the neutral hypothesis explained the observed data well in $8 \%$ of the cases [7]. However, $44 \%$ of the studies matched traditional niche partitioning models, whereas the remaining studies either matched mixed models or were not assigned. Thus, niche factors appear to be

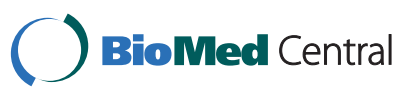


essential in many cases for explaining biodiversity but the integration of stochastic elements may improve interpretation. Most research addressing these hypotheses has been performed with plants and animals. For fungi, such research has focused on arbuscular mycorrhizal (AM) fungi, which are widespread root symbionts of a vast range of plant species. AM fungi promote host nutrition, diversity and survival under biotic and abiotic stress conditions $[8,9]$. Besides AM fungi, other types of fungal mutualists, for example endophytes, can improve the health and the performance of plants. Studies on endophytes have assessed their occurrences and their influences on their hosts and on plant community structure [10-13]. However, further research is required to elucidate the causes and mechanisms leading to the observed diversity of endophytes.

Common reed (Phragmites australis (Cav.) Trin. ex Steudel) has been used as a model to investigate the interactions of a plant with its associated mycoflora and the interactions between different fungi colonizing the same host. Previously, it was found that many different fungi colonized healthy common reed growing in the native freshwater habitats of Lake Constance in the northern alpine forelands of Germany. The number of fungal species identified by cultivation-independent, molecular approaches $[14,15]$ clearly exceeded those isolated by classical cultivation $[16,17]$. However, only a fraction of the many fungal species present reached a high prevalence, suggesting that competition and niche differentiation may shape these communities.

Abiotic and biotic factors, which distinguish various niches and which may allow some fungal species to dominate over others, are manifold. One approach to identify such factors is to analyze distribution patterns of fungal species observed in classical cultivation schemes, in gene libraries from cloned environmental DNA or in datasets generated using other molecular approaches. The need for sufficient replications in such studies can be met by employing nested-PCR assays that monitor specific fungal species in large collections of field samples. For common reed in Lake Constance these analyses revealed that habitat type and host organ influenced the occurrences of two uncultured fungi [15]. Additional abiotic and biotic factors that may lead to niche differentiation like temperature, $\mathrm{pH}$, carbon, nitrogen, and other resources can be analyzed, if cultured strains are available.

Isolates belonging to the genus Microdochium (Ascomycota, Pezizomycotina, Sordariomycetes, Xylariales), were the most frequent among those recovered from $P$. australis under conditions favoring the isolation of endophytes [16]. These Microdochium isolates were preliminarily assigned to Microdochium sp. and $M$. phragmitis. The former, which was later characterized as $M$. bolleyi, was shown to colonize living roots of reed without causing symptoms [18]. M. bolleyi has a broader host range, since it occurs as a minor root pathogen or an endophyte on other grasses as well [19-21]. $M$. phragmitis seems, however, to associate only with reed.

To investigate coexistence, several approaches were used to search for evidence of niche partitioning between fungal species sympatrically colonizing common reed at Lake Constance. Presence-absence patterns were obtained using specific nested-PCR assays on a large set of field samples determining co-occurrences of the two Microdochium species and three additional, unrelated species. Furthermore, whether divergent growth temperature optima and resource partitioning could define the niches of the two closely related fungal species was examined.

\section{Methods}

\section{Cultivation of fungi}

The fungal isolates used in this study (Additional file 1) originated from a previously published study [16]. Reference strains were purchased from CBS (Utrecht, Netherlands). All fungi were cultured on $2 \%$ malt agar (Biomalt, Villa Natura Gesundprodukte GmbH, Kirn, Germany) at $20^{\circ} \mathrm{C}$ in the dark.

Mycelial growth rates were determined using three culture replicates for each isolate and each temperature assayed. These ranged from $0^{\circ} \mathrm{C}$ to $30^{\circ} \mathrm{C}$ at intervals of $5^{\circ} \mathrm{C}$. The mycelial radii for all cultures were determined after $14 \mathrm{~d}$ and additionally at $7 \mathrm{~d}$ for cultures incubated at temperatures ranging from $15^{\circ} \mathrm{C}$ to $30^{\circ} \mathrm{C}$. Four individual isolates were analyzed for the 5/97-16 sequence type and five isolates for the 5/97-54 sequence type. Two reference strains were used for $M$. bolleyi (CBS 137.64, CBS 172.63), and for M. nivale (CBS 110.94, CBS 320.78), respectively. Where applicable, data from strain replicates were combined and averaged. The data were analyzed statistically using the Dunnett test and multifactorial analysis of variance (MANOVA) that separately analyzed the growth rates of the isolates belonging to a species and their individual replicates (confidence limits at $P<0.05)$. Both tests were implemented using JMP software version 4.04 (SAS Institute, Cary, NC, USA).

\section{DNA extraction, PCR, sequencing and phylogenetic analysis}

DNA preparations from fungal mycelia were performed as described previously [22]. DNA preparations from reed tissues used for nested-PCR assays had been conducted earlier $[17,22]$ and were kept frozen at $-20^{\circ} \mathrm{C}$. Reed was harvested from Lake Constance (Germany) at four sites, described previously [16]. 
DNA sequences of the ITS (internal transcribed spacers) rDNA region from fungal isolates were produced, assembled, aligned and edited as previously described [22].

Phylogenetic analysis relied on the alignment of 37 sequences created using the software ClustalX ftp://ftp. ebi.ac.uk/pub/software/mac/clustalx and then manually adjusted. The alignment comprised the ITS1-box, the $5.8 \mathrm{~S}$ rRNA gene, and the ITS2-box. Besides the sequences of isolates listed in Additional file 1 sequences from related fungi were included that were detected using BlastN searches in public databases. The program PAUP Version $4.0 \mathrm{~b} 10$ was used to generate the phylogenetic tree depicted in Figure 1[23]. The BioNJ method with the HKY85 setting for distance measures was used to create a tree that served to estimate the proportions of invariable sites and gamma shapes. A heuristic search under the maximum likelihood criterion and the GTR+G+I substitution model, using the neighbor-joining tree as input, was created. The confidence of the resulting ML tree was estimated using 1000 quartet puzzle steps.

Sequences obtained during this study were deposited in the EMBL-EBI Nucleotide Sequence Database (European Molecular Biology Laboratory-European Bioinformatics Institute; http://www.ebi.ac.uk/) under the accession numbers AM502255 to AM502266 (Additional file 1).

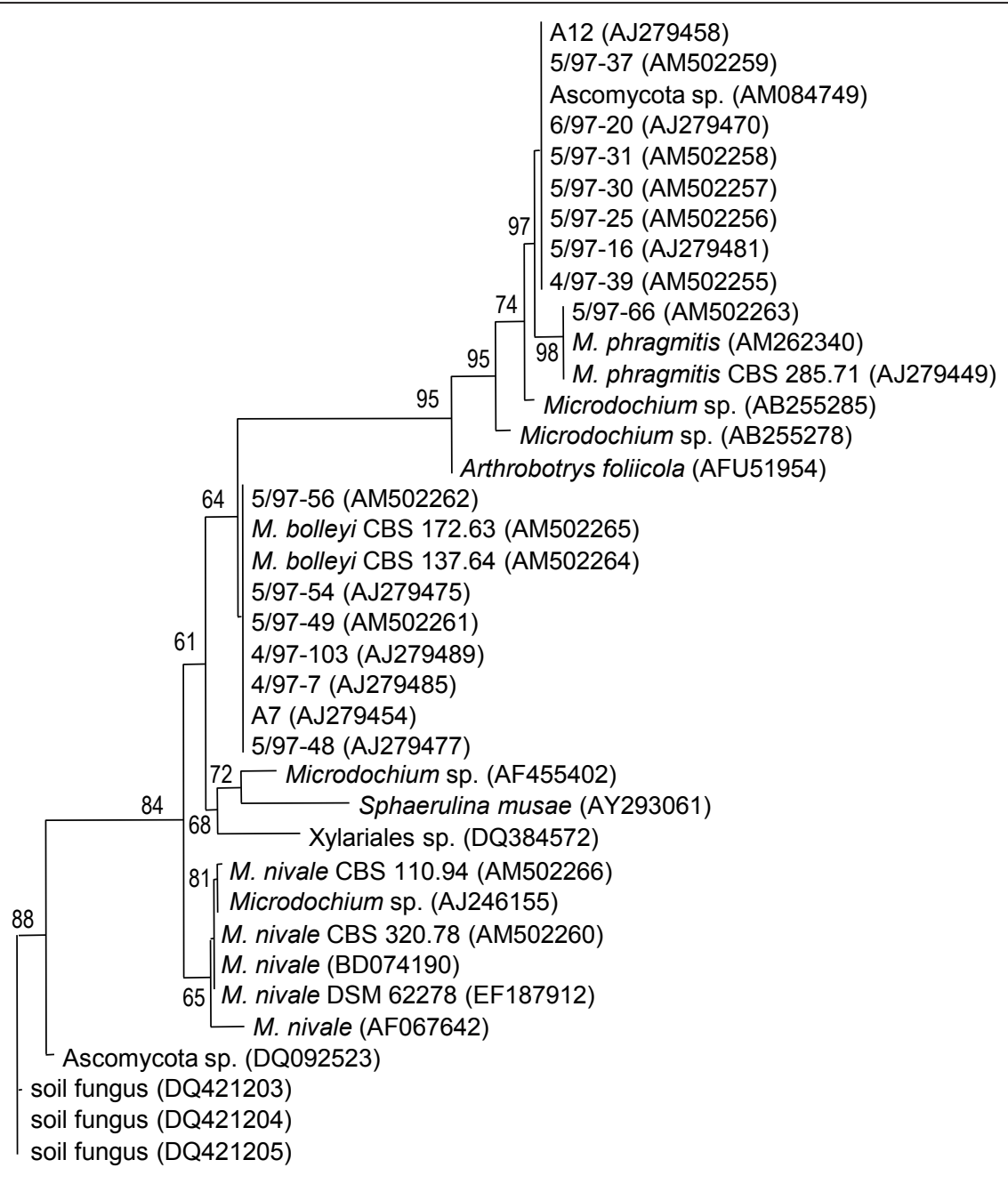

0.1

Figure 1 Molecular phylogeny of Microdochium spp. Molecular phylogeny obtained using Maximum Likelihood analysis on ITS rDNA, displaying the relationships between 37 sequences originating from reed isolates, their closest database matches, as well as additional references. Accession numbers are provided in brackets. Reference sequences are shown as annotated in the source database. Support of branches is shown when higher than $50 \%$. 


\section{Nested-PCR assays}

DNA preparations originated from 251 samples of 66 standing reed plants that were harvested from Lake Constance from July 1998 to August 2001 [17]. The same DNA preparations had been used earlier to determine the distribution of three additional fungi that were frequently observed in common reed using specific nested-PCR assays [15,17]. These previous data allowed assessment of fungal co-occurrence at a broader scale investigating whether other fungi may have influenced the prevalence of Microdochium spp. Two of the additional fungi were uncultured Ascomycota and were originally identified using a molecular approach [15]. They were designated as Ms7Mb4 (related to Podospora) and Ms43Mb21 (distantly related to an uncharacterized ericoid mycorrhizal fungus). The third fungus was an endophyte, Stagonospora sp. 4/99-1 that originated from cultivation [17].

The first PCR-step of the two-step nested-PCR assay targeted the Eumycota using the primers ITS1F and ITS4. Reaction mixtures contained: 100 ng of DNA, 2 $\mathrm{mM} \mathrm{MgCl}_{2}, 0.2 \mathrm{mM}$ dNTPs, $0.5 \mathrm{mg} / \mathrm{mL}$ bovine serum albumin, $0.25 \mu \mathrm{M}$ of each primer and $0.05 \mathrm{U} / \mu \mathrm{L}$ of recombinant Taq DNA Polymerase (MBI Fermentas) in a total volume of $25 \mu \mathrm{L}$. An initial denaturation step at $94^{\circ} \mathrm{C}$ for $150 \mathrm{~s}$ was followed by 40 cycles of the following protocol: $94^{\circ} \mathrm{C}$ for $30 \mathrm{~s}, 55^{\circ} \mathrm{C}$ for $15 \mathrm{~s}$ and $72^{\circ} \mathrm{C}$ for $45 \mathrm{~s}$ plus one additional second per cycle. The reaction was terminated by a final extension at $72^{\circ} \mathrm{C}$ for $10 \mathrm{~min}$. The second PCR step applied specific primers annealing at the highly variable ITS1 and ITS2 boxes. These primers were: 5/97-54/ITS.F2 (5'-GGT GCT GGA AAC AGT GCT GCC AC-3') and 5/97-54/ITS.R2 (5'-GTC GTC TGG CCG GCT TGC AG-3') that were derived from the sequence of isolate $M$. bolleyi 5/97-54 (Accession no. AJ279475), and 5/97-16/ITS.F2 (5'-ACC CGA AAG GGT GCT GGA AG-3') and 5/97-16/ITS.R2 (5'TTG GCT ATC GTC TAG ACG TGT TCA A-3') that were derived from the sequence of $M$. phragmitis 5/9716 (Accession No. AJ279481). Reaction mixtures contained: $0.25 \mu \mathrm{L}$ of the first PCR reaction, $1.5 \mathrm{mM}$ $\mathrm{MgCl}_{2}, 0.2 \mathrm{mM}$ dNTPs, $0.5 \mathrm{mg} / \mathrm{mL}$ bovine serum albumin, $0.125 \mu \mathrm{M}$ of each primer and $0.05 \mathrm{U} / \mu \mathrm{L}$ of recombinant Taq DNA Polymerase in a total volume of $25 \mu \mathrm{L}$. Reactions with primers 5/97-54/ITS.F2 and 5/97-54/ITS. $\mathrm{R} 2$ included an initial denaturation step of $94^{\circ} \mathrm{C}$ for 120 $\mathrm{S}$ that was followed by 5 cycles of a touch-down proto$\operatorname{col}\left(94^{\circ} \mathrm{C}\right.$ for $30 \mathrm{~s}, 82^{\circ} \mathrm{C}$ for $45 \mathrm{~s}$ with a decrease of $1^{\circ} \mathrm{C}$ per cycle) and then by 40 additional cycles $\left(94^{\circ} \mathrm{C}\right.$ for 30 $\mathrm{s}, 77^{\circ} \mathrm{C}$ for $45 \mathrm{~s}$ plus one additional second per cycle). This was followed by a final extension at $77^{\circ} \mathrm{C}$ for 10 min. Reactions with primers 5/97-16/ITS.F2 and 5/9716/ITS.R2, basically followed the same scheme but had an initial annealing temperature of $77^{\circ} \mathrm{C}$ at the first cycle, followed by a touch-down to $72^{\circ} \mathrm{C}$. Positive and negative controls included genomic DNAs of target and non-target fungi, respectively.

Results of nested-PCR assays were scored as 0 vs. 1 and statistically analyzed using a contingency table and a binomial distribution test $(P<0.05)$ with the Bonferroni correction. The co-occurrences of two fungi in the same samples were examined using pair-wise contingency analysis and two-sided Fisher's Exact test (confidence limits at $P<0.05$ ) to determine deviation from a random distribution, either positive or negative. Fisher's Exact test provides a precise likelihood for the observed distribution, but is restricted to pair-wise analysis. These statistical analyses were performed using JMP version 4.04. Analyses of co-occurrences of several species were carried out with the Co-occurrence module in the software EcoSim Version 7.72 http://garyentsminger.com/ ecosim/index.htm. EcoSim applies a Monte Carlo approach to create a random distribution of data for statistical testing that is compared to the experimental data to test the null hypothesis that the co-occurrence patterns observed in the field samples result from random variation (confidence limits at $P<0.05$ ) [24]. The recommended default settings were used except for the number of randomized data matrices generated by the software, which was increased to 10000 . It had previously been suggested that deviation from other default program settings, that keep the number of species observed in each sample ("fixed columns") constant, as well as the sum of the incidences of each species ("fixed rows") for the randomizations, could result in misleading assertions [25].

Canonical correspondence analysis (CCA) with PCORD version 5.10 (MjM Software Design, Gleneden Beach, OR) was used to assess the degree to which each of three factors analyzed contributed to the frequencies of the fungi detected in the field samples. CCA used the incidences of five species examined for 46 distinct habitat-organ-month combinations. In CCA setup, axis scores were standardized using Hill's method. Axes were scaled to combine representation of species and stands. Stand scores were treated as linear combinations of factors. Graph ordination was set up in two dimensions to present the two most important factors. A Monte Carlo permutation test based on 999 random permutations was applied to test the null hypothesis that the community was independent of the analyzed factors.

\section{Registration of substrate utilization spectra}

Microdochium isolates were grown in liquid medium containing $4 \mathrm{~g} / \mathrm{L}$ glucose, $10 \mathrm{~g} / \mathrm{L}$ malt extract, $4 \mathrm{~g} / \mathrm{L}$ yeast extract [26] for $3-7 \mathrm{~d}$ at $20^{\circ} \mathrm{C}$ and $120 \mathrm{rpm}$ to obtain the inoculum for the physiological tests. The reed strains A7, 4/97-7, 5/97-48, 5/97-49, and 5/97-54 
were taken for $M$. bolleyi, whereas 4/97-39, 5/97-16, 5/ 97-30, and 6/97-20 represented M. phragmitis. In addition, reference strains from CBS, (Additional file 1) were analyzed. Mycelia were harvested using filtration, washed with autoclaved distilled water and re-suspended in $2 \%$ carrageenan type II (Sigma, Deisenhofen, Germany) to provide an OD590 of 0.05. Each well of a BIOLOG SF-N2 plate (Merlin Diagnostika GmbH, Bornheim, Germany) was inoculated with $100 \mu \mathrm{l}$ of mycelial suspension. These microtiter plates contained 95 different carbon sources and one control well without any carbon source. Plates were incubated for $10 \mathrm{~d}$ at $21^{\circ} \mathrm{C}$ in the dark. Thereafter, absorption at $560 \mathrm{~nm}$ was recorded using an ELISA reader (SLT Spectra, SLT Laborinstrumente GmbH, Grödig, Austria). After exporting the data to Microsoft Excel, the absorption in the control well was defined as $0 \%$ growth and that of the well with the maximum absorption as $100 \%$. All other values were scaled in relation to these limits. For each isolate tested, three independent experiments were performed and the transformed results were averaged. The t-test and the Dunnett test in JMP were used to assess the variation between species for each carbon source using the average values of each isolate (confidence limits at $P<0.05$ ). Furthermore, the overall similarity of carbon usage patterns between species was compared using the Niche Overlap module in EcoSim [24]. All four EcoSim randomization algorithms (RA1RA4) were used to generate 10000 simulated data matrices in each case. For all other parameter settings, the default was used.

\section{Results}

\section{Molecular characterization of Microdochium isolates}

A molecular phylogeny of the ITS region was generated that included previous sequences from Microdochium spp. [16], new sequences from Lake Constance reed isolates and from CBS reference strains (Additional file 1), and in addition, sequences from databases that were identified by BlastN searches. The phylogeny indicated the existence of three different sequence variants among the isolates colonizing common reed at Lake Constance (Figure 1). Eight isolates had identical sequences and were typified by the previously described isolate 5/97-16 [16]. This sequence variant had $98.4 \%$ identity to the reference $M$. phragmitis (CBS 285.71). A single isolate, 5/97-66, was identical to CBS 285.71. We treated all these isolates as M. phragmitis. This degree of similarity was clearly higher than the limit of $97 \%$ that had previously been suggested to differentiate fungal species using their ITS sequence $[27,28]$. Furthermore, because intraspecific variation in the rRNA gene cluster is known in eukaryotes including fungi, a higher threshold value may introduce the risk of wrongly dividing isolates belonging to a single species into different species. A previous study found that intraspecific ITS variation ranged from 0.16 to $2.85 \%$ in Ascomycota and Basidiomycota [29]. Another group of seven isolates had sequences that formed a cluster with the references $M$. bolleyi CBS 137.64 and CBS 172.63 . They diverged by at most $0.5 \%$ from each other. Therefore, and because typical morphological characters were highly similar compared to these references (data not shown), the previously described Microdochium sp. typified by isolate 5/97-54 [16] was treated here as M. bolleyi. None of the isolates from reed clustered with references belonging to $M$. nivale or any of the other species included in the phylogram.

\section{Nested-PCR assays indicate niche differentiation of Microdochium spp}

To examine whether colonization of $P$. australis by the two species of Microdochium reflected stochastic patterns or niche differentiation two nested-PCR assays were designed that specifically targeted the ITS sequence of the 5/97-16 and of the 5/97-54 sequence variants. The specificities of these assays were tested using genomic DNA preparations as templates that were extracted from the fungal isolates typifying the Microdochium sequence variants identified above and from additional isolates belonging to other genera of Ascomycota that had been recovered from $P$. australis earlier [16]. Genomic DNA from aseptically grown $P$. australis served as an additional negative control. As anticipated, the first PCR step, which used standard primers targeting the Eumycota, yielded reaction products with all fungal templates (Additional file 2A). The second PCR steps using primers directed against the individual Microdochium species yielded reaction products only with DNA from the targeted fungi (Additional file 2B-C).

The incidences of the two Microdochium species in 251 DNA samples covering a period of three years, four host organs, i.e. rhizome, root, stem, and leaf, and two contrasting habitat types, i.e. flooded and dry, were analyzed. Both targets were generally detectable in all organs, at all sites and throughout the seasons. The overall detection frequency was $22 \%$ for $M$. phragmitis and $27 \%$ for $M$. bolleyi. From each nested-PCR series one randomly chosen product for each host organ was sequenced to validate the correctness of the method employed. Each of the eight PCR-products corresponded to the respective previously determined sequences (data not shown).

In general, the results from the nested-PCRs on the field samples indicated for both targets, but especially for $M$. phragmitis, a reduced prevalence during the warm summer months when the data were pooled 
across host habitat and host organ (Figure 2). Statistical support for this observation was obtained for M. phragmitis when comparing its minimum, i.e. July, in a pairwise manner with the other months that demonstrated a significant difference to April (binomial test, $P=$ $0.006)$ and November $(P=0.007)$. In addition, the variance between September and November was also significant $(P=0.007)$. When applying the stringent Bonferroni corrections on an analysis testing all months against each other, all variations appeared non-significant. Variations in the corresponding data for the other target, $M$. bolleyi, did not show any significance, neither when analyzed in a pair-wise manner nor in a total analysis. For both targets, there was no statistical support for seasonal variation when evaluating the results for the individual host organs separately (data not shown, binomial test with $P<0.05$, Bonferroni corrected). When comparing the detection frequencies of the two fungi against each other none of the apparent variations proved to be significant for any month when the data were pooled across organs (binomial test with $P<0.05$, Bonferroni corrected) (Figure 2).

Statistical analysis of variation with respect to the colonized host organ revealed for both, $M$. phragmitis and M. bolleyi, a significant preference for roots (binomial test with $P<0.05$, Bonferroni corrected). Besides host organ, also the host habitat affected the incidences of the fungi. M. phragmitis occurred significantly more frequently at flooded sites compared to dry sites $(27 \%$ vs. $16 \%$, binomial test, $P=0.0385$ ) when the data were pooled across organ. The opposite result was obtained for M. bolleyi ( $19 \%$ vs. $34 \%$, binomial test, $P=0.0110)$. When examining variation resolved for all host organhabitat type combinations (Figure 3, small letters), $M$. phragmitis showed a significant preference for roots at flooded sites $(P=0.0127)$, whereas $M$. bolleyi significantly preferred roots $(P=0.0002)$ and in addition rhizomes $(P=0.0386)$ at the dry sites. Comparisons between the two species showed that roots were the only organs with significantly contrasting preferences for the habitat type (root-flooded: $P=0.0213$; root-dry: $P=$ 0.00004) (Figure 3, capital letters).

\section{Carbon utilization patterns of Microdochium spp}

To determine whether resource partitioning, as a biotic attribute, may have contributed to these findings the potential of Microdochium spp. to utilize 95 different carbon sources was tested in vitro. The EcoSim Niche Overlap module was used to evaluate the overall similarity in carbon usage. The niche overlap index in the experimentally obtained data set was 0.9733 , whereas the mean of the simulated matrices was 0.7127 , using default parameters for calculation (RA3 model). This difference was statistically significant $(P<0.05)$, and thus indicated that the carbon usage of the two species was overall more similar than expected by chance. The application of alternative parameters for the calculation (i.e. the RA1, RA2, and RA4 models) led to the same conclusion. In addition, intra-species comparison of different strains belonging to the same species showed that within each of the two species there were significantly more resource overlaps than expected by chance (data not shown).

Although the carbon utilization capabilities of the two species were similar, specific differences existed, which were statistically assessed using t-tests. Significant differences between the two species $(P<0.05)$ were observed for 21 substrates (22.1\%) (Additional file 3). In addition,

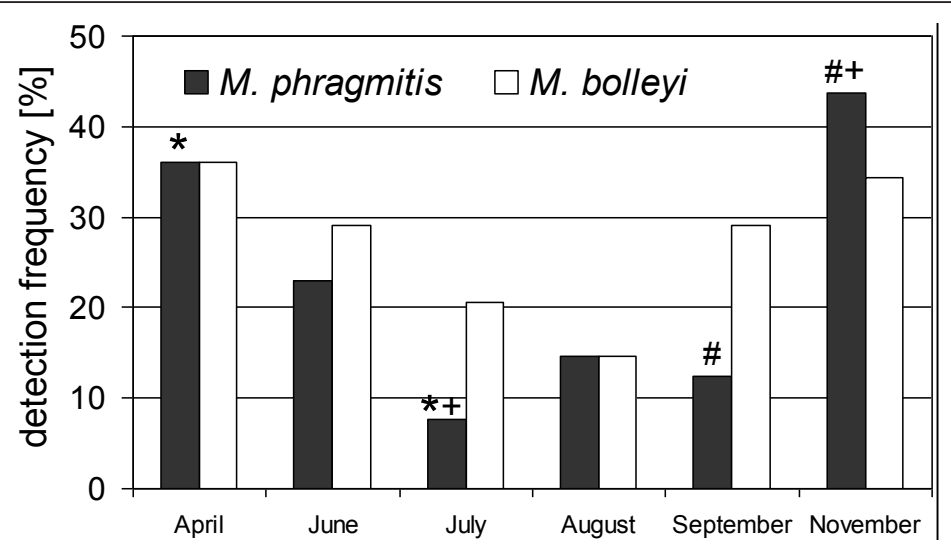

Figure 2 Seasonal variation of Microdochium spp. on Lake Constance reeds. Summary of nested-PCR assays on 251 DNA preparations from tissue samples of $P$. australis harvested over a period of three years. Detection frequency for each target shows the percentage of samples producing a band after the second step of the nested-PCR. Results from all sites and all host organs were pooled. Symbols on top of the columns indicate significant variation between the respective months when analyzing each fungus separately (binomial test with $P<0.05$ ). Occurrences of $M$. phragmitis differed significantly when comparing April with July $(*)$, July with November (+), and September with November (\#). 


\section{M. phragmitis $\square$ M. bolleyi}

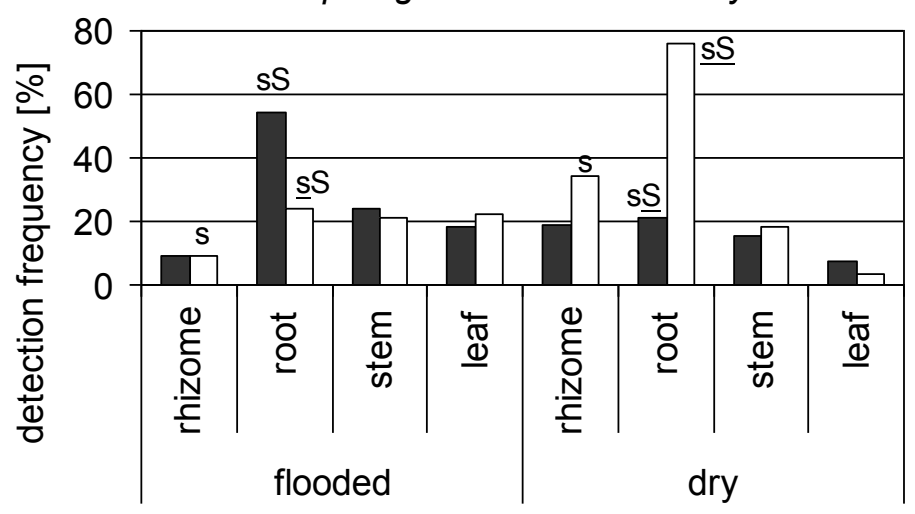

Figure 3 Habitat preferences of Microdochium spp. on Lake Constance reeds. Summary of nested-PCR assays on 251 DNA preparations from tissue samples of $P$. australis. Detection frequency for each target shows the percentage of samples producing a band after the second step of the nested-PCR. Results from all seasons were pooled. Small letters compare variation between the two habitat types when analyzing each target species and each host organ separately (binomial test with $P<0.05$ ). Capital letters compare variation between the two species when analyzing each host organ and each habitat separately (binomial test with $P<0.05$ ). $\mathrm{S} / \mathrm{s}$, variation is significant; non-significant variation is not indicated. Underlined letters indicate that the variation remains significant after Bonferroni correction.

the application of the Dunnett test rendered essentially the same results (not shown). M. bolleyi grew significantly better than M. phragmitis on 10 of the 95 carbon sources tested (Additional file 3). Conversely, M. phragmitis grew significantly better than $M$. bolleyi on 11 carbon sources (Additional file 3).

\section{Temperature ranges for growth of Microdochium spp}

The potential effect of temperature, as an abiotic attribute, was tested to determine if it could distinguish these fungi and explain their observed distributions in field samples. All reed isolates of each species exhibited highly similar growth rates at all temperatures tested. $M$. bolleyi isolates had a maximum at $25^{\circ} \mathrm{C}$ and were still able to grow at $30^{\circ} \mathrm{C}$ (Figure 4). M. phragmitis isolates had growth rates a little higher than those of $M$. bolleyi up to $20^{\circ} \mathrm{C}$. However, they grew much more slowly above $20^{\circ} \mathrm{C}$, which was their maximum, and they completely ceased to grow at $30^{\circ} \mathrm{C}$. This also applied to M. nivale, which was used as an outgroup. When such cultures were transferred to a temperature of $15^{\circ} \mathrm{C}$ the colonies started to expand again, indicating that these fungi were still viable. Dunnett tests $(P<$ 0.05 ) on the averaged growth rates of the $M$. phragmitis and M. bolleyi isolates indicated significant differences for all temperatures tested except $20^{\circ} \mathrm{C}$. Multifactorial analysis of variance (MANOVA, $P<$ 0.05 ) yielded the same result.

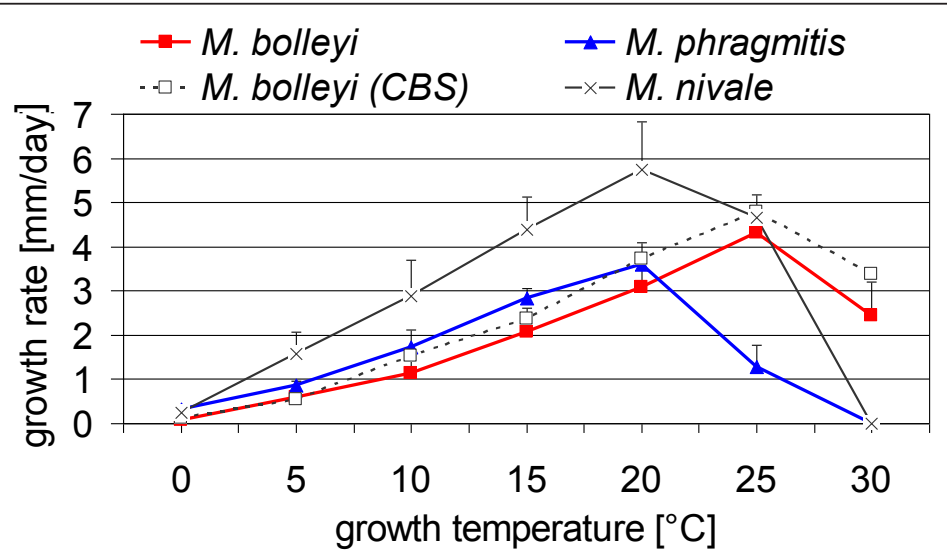

Figure 4 Growth rates of Microdochium spp. Three replicated assays were recorded for each isolate and each temperature. For each taxon up to five independent isolates were tested. Results show total averages from all replicated assays and all isolates. The tested reed isolates were for M. phragmitis 4/97-39, 5/97-16, 5/97-30, and 6/97-20, and for M. bolleyi A7, 4/97-7, 5/97-48, 5/97-49, and 5/97-54. The tested reference strains were for M. bolleyi CBS 172.63 and CBS 137.64, and for M. nivale CBS 320.78 and CBS 110.94. Bars indicate standard deviations. 
Whether temperature optima determined in vitro could correspond to soil temperature at sites where the fungi were originally isolated was investigated. Three data loggers were buried at a soil depth of $20 \mathrm{~cm}$ at one of the original locations on the shores of Lake Constance to record hourly ambient temperatures from March 2005 to February 2006 (K. Neubert \& J. Nechwatal, data not shown). The average annual soil temperature was $11.1^{\circ} \mathrm{C}$ with a maximum reaching slightly above $21^{\circ} \mathrm{C}$, which occurred for only a few days in summer. Both species grew equally well in vitro at $21^{\circ} \mathrm{C}$.

\section{Co-occurrences of five fungal species}

Four statistical tests were used to analyze the occurrences of five fungi, i.e. the binomial distribution test, the EcoSim Co-occurrence module [24], Fisher's Exact test and Canonical Correspondence Analysis (CCA). Except for CCA, one combined data set, including nested-PCR results from all 251 samples, was analyzed simultaneously using each method, and in addition, several partial data sets were analyzed to examine occurrences for season, host organ, host habitat, and for the combination of organ plus habitat, respectively.

First, the binomial distribution test $(P<0.05)$ with Bonferroni corrections was applied to examine whether within a given data set the total incidence of one species differed significantly from that of another species. Three of ten species pair comparisons using the undivided data set showed significantly contrasting occurrences (Additional file 4). These three comparisons involved Ms43Mb21, which was generally less prevalent than all other species. Although season did not significantly separate the species pair M. phragmitis - M. bolleyi (as mentioned above), the inclusion of the three additional species showed that this factor contributed to the separation of the five species. Four of 60 species pair comparisons $(6.7 \%)$ using data sets divided by months (ten species pairs, six months) showed significant differences (Figure 5A, Additional file 4). Nine of 40 species pair comparisons (22.5\%) using data sets divided by host organ showed significant differences (Additional file 4). Five of 20 species pair comparisons (25\%) using data sets divided by habitat type showed significant differences (Additional file 4). Ten of 80 species pair comparisons $(12.5 \%)$ using data sets divided by the combination of organ plus habitat showed significant differences (Figure 5B, Additional file 4).

The second statistical test was the Co-occurrence module of EcoSim. In a total data set comprising all five species, significantly less co-occurrence was observed compared to the null hypothesis $(P<0.05$; data not shown). The analyses of data matrices that reflected the distributions of the five species in the individual months exhibited significantly decreased co-occurrences in
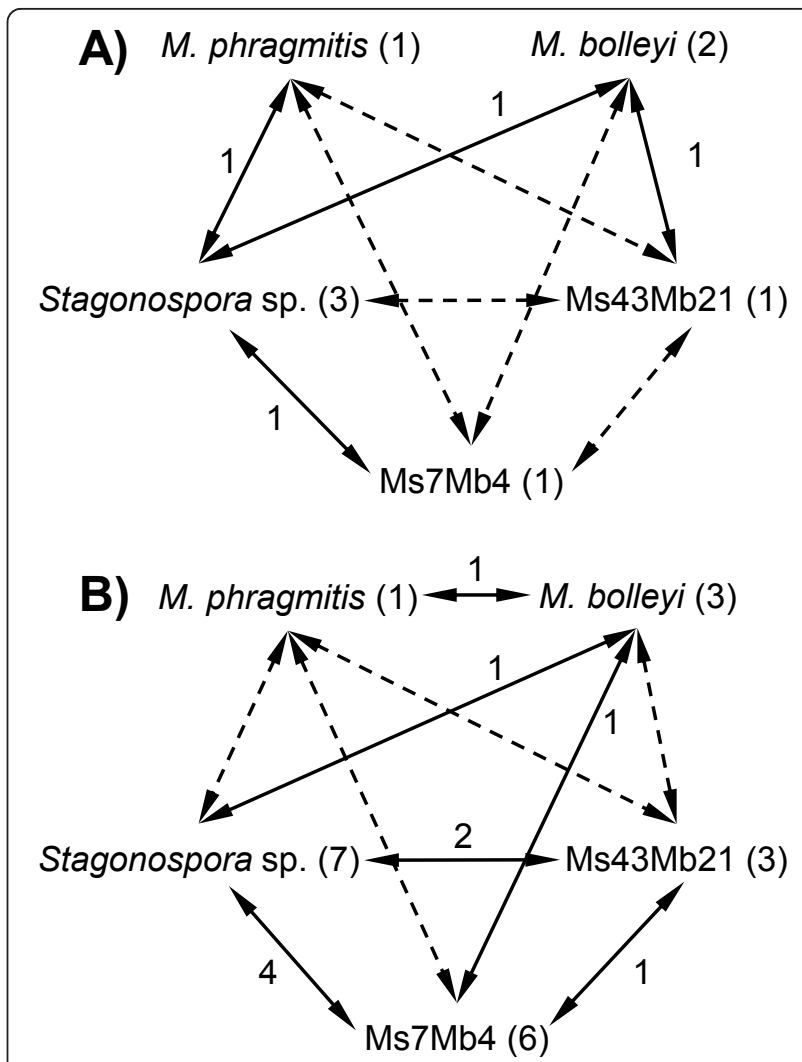

Figure 5 Niche differentiations of five fungal species with respect to time and space. Summary of nested-PCR assays on 251 DNA preparations from tissue samples of $P$. australis. Pair-wise species comparisons were conducted using binomial tests with $P$ $<0.05$. Straight arrows indicate variations that remained significant after Bonferroni corrections, broken arrows variations that were additionally significant when Bonferroni corrections were omitted. Numbers at the arrows give the incidences of significant results for a species pair and those in brackets for a given species, respectively. Numbers refer to Bonferroni-corrected comparisons. A) Seasonal variation by months; B) Spatial variation by host organ plus habitattype.

August and September. Accordingly, assessment of individual organs demonstrated significantly decreased cooccurrences for stem. Both habitats surveyed, dry, and flooded, showed significantly decreased co-occurrences. From the eight organ-habitat combinations, only stems from the dry habitat exhibited a significant decrease. We did not observe a significant increase of co-occurrence in any of the analyses.

The third statistical test applied was Fisher's Exact test $(P<0.05)$ with Bonferroni corrections to determine if certain species pairs may co-occur significantly more or less frequently in the same samples than expected by chance. Three of ten species pair comparisons (M. bolleyi vs. Ms7Mb4 and vs. Ms43Mb21, respectively, and Ms7Mb4 vs. Ms43Mb21) using the undivided data set showed significantly more co-occurrences (Additional 
file 5). Only the pairing of Stagonospora sp. vs. Ms7Mb4 co-occurred less frequently than expected by chance. Neither the analyses of the 60 species pair comparisons using data sets divided by months nor those of the 40 comparisons using data sets divided by host organ showed any significant differences when applying Bonferroni corrections (Additional file 5). In contrast, examination of data sets separated for host habitat revealed that M. bolleyi co-occurred with Ms7Mb4 and Ms43Mb21 more frequently at the dry habitat than expected by chance. Under the same conditions, M. bolleyi co-occurred with Stagonospora sp. less frequently. None of the 80 pair-wise species comparisons that examined data sets divided by the combination of organ plus habitat showed significantly increased or decreased co-occurrences (Additional file 5).

Finally, CCA was used to estimate which of the analyzed factors most influenced the occurrences of five species in all samples analyzed. Space at the level of organ explained $32.9 \%$ of the observed total variation, whereas space at the level of habitat and time at the level of months did so for $5.5 \%$ and $0.1 \%$, respectively. A plot including the two main axes indicated that all five species were well separated for at least one factor (Figure 6). It underlined that Stagonospora sp. was distinguished from the other species mainly because of its distinct organ preferences. The CCA plot confirmed that habitat type was most important for separation of the two Microdochium species. For the remaining species, both organ and habitat determined their separation.

\section{Discussion}

Previous studies have indicated that fungal endophytes may coexist at very small scales. In this study, niche partitioning between two endophytic species of Microdochium sympatrically colonizing Phragmitis australis was assessed. M. bolleyi and M. phragmitis were found to be significantly segregated for host habitat, but not for host organ and season. However, when additional, unrelated fungi that colonize the same host were also included in the analyses, the latter two factors were also found to contribute to niche partitioning.

Several factors can cause niche differentiation between endophytes, which may attenuate competition and thus allow for a high fungal diversity on the same host species. One factor is space, which is with respect to endophytes hierarchically structured from continent to region, to habitat, to host individual, to host organ, and further down to the level of host cells. Two of these levels, i.e. the habitat type and the host organ, were analyzed. Both, M. bolleyi and M. phragmitis, preferentially colonize the same organ, i.e. roots, confirming an earlier result [16]. Within the limits of detection, nested-PCR assays in this study indicated that $M$. bolleyi occurs more frequently on roots at dry sites, whereas $M$. phragmitis occurs more frequently on roots at flooded sites. This suggests spatial niche partitioning at the level of habitat type. Like all molecular assays employing fungal genomic DNA extracted from field samples, the assays from this study cannot distinguish between growing and dormant cells, and thus cannot provide details on metabolic activities or developmental stages. In addition, a possible introduction of bias against rare templates during the first stage of the nested-PCR has to be considered, which would produce false-negative results in case of fungi present at very low abundance. However, if the first step of nested-PCR comprises as many cycles as used here rare templates will be over- not under-amplified, as previously shown [30]. Thus, for assessment of presence-absence data nested-PCR is a highly specific and sensitive method.

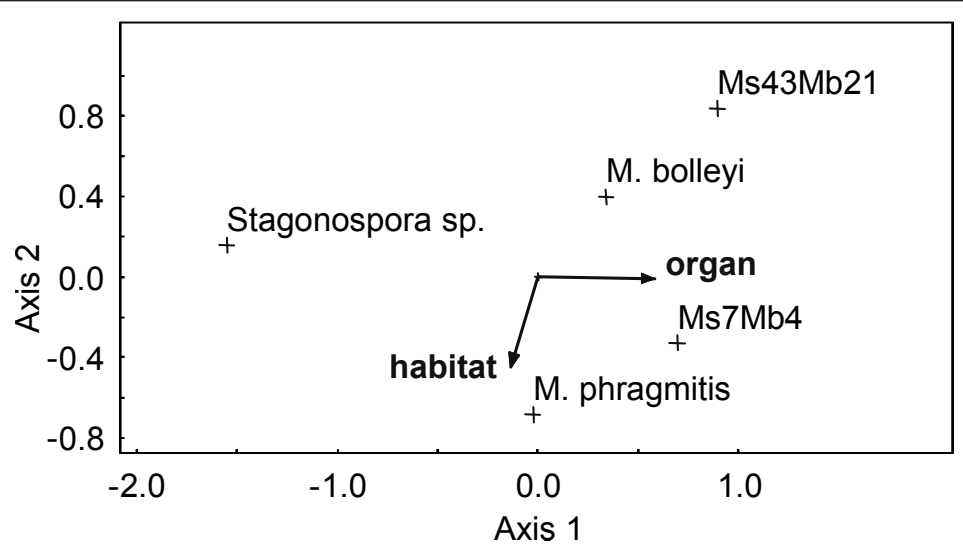

Figure 6 Canonical correspondence analysis. CCA biplot ordination for the effects of space defined by plant organ and habitat type assessing five fungal species on reeds at Lake Constance. Axes 1 and 2 explain 32.9\% and 5.5\% of the variation, respectively. Monte Carlo permutation test on axis 1: $P=0.0010$. 
Further support for an influence of spatial niche partitioning on the composition of the reed-associated fungal community was obtained when occurrences of three additional species were also considered. Both binomial tests and CCA indicated that all five species were differentiated by host organ and / or habitat. Since P. australis has a vast geographical distribution, it would be interesting to assess the factor space in structuring fungal communities at higher hierarchical levels in the future.

The importance of space in affecting fungal community composition has previously been acknowledged. Much of this information comes from pathogens of agronomically important crops [31] and from mycorrhizal fungi [14,32-36]. In addition, endophyte communities seem also to be influenced by the factor space [37-39]. However, in contrast to other types of fungi, little is known about the causes leading to spatial differentiation in endophytes. At the same sites examined in this study an even more distinct preference for the habitat type was previously noted for AM fungi that were not observed at flooded sites at all, whereas at the dry sites, 21 phylotypes were detected at various frequencies [14]. Vertical distribution patterns of reed-associated fungi have been recorded in a brackish tidal marsh, with diverse communities depending on the leaf layer [40]. Site-dependent differences in reed stands are known for Oomycota, where some species preferred either dry or flooded sites [41]. It seems likely that it is not space per $s e$, but rather specific physico-chemical features of the respective sites that cause such differences.

Another factor that can cause niche differentiation between fungal endophytes is time, resolved here at the scale of individual months of the season. The progress of the season drives host developmental processes like the emergence of shoots and leaves in spring and senescence in autumn, and thus dynamically modifies the niches available to plant-associated fungi. The occurrences of $M$. bolleyi and $M$. phragmitis were similar for season. Thus, seasonal niche partitioning does not seem to significantly separate Microdochium spp. on common reed. However, binomial tests indicated that time was a factor involved in the separation of the five species analyzed.

The contribution of seasonal variation to fungal community variation has previously been recognized. Endophytic colonization of tropical cacao trees increased with leaf age and partially protected the host against pathogenic Phytophthora sp. [42]. Similarly, endophytic diversity increased during leaf development in Camellia japonica, whereas epiphytic diversity remained stable with season [43]. Seasonal succession was also demonstrated for the mycoflora in a Colorado mountain soil that changed substantially between spring and summer, suggesting functional differentiation [44]. Seasonal variation has been found in an aquatic fungal community decomposing plant debris in streams [45]. In reed stands at Lake Constance, Oomycota populations were shown previously to exhibit seasonal variation [46]. For the reed pathogen Pythium phragmitis, minimal detection in August resembled the decrease of Microdochium spp. during the summer.

Temporal niche differentiation thus contributes to the separation of the five species examined, although to a lesser extent than space. Thus, niche differences resulting from abiotic or biotic attributes seem to separate these fungi and may explain their coexistence on the same host. Temperature was one attribute that distinguished the two Microdochium species in vitro. M. phragmitis, which occurs more frequently at flooded sites, grows faster at lower temperatures, whereas $M$. bolleyi, which prefers dry sites, grows faster at higher temperatures. For most of the year, based on the in vitro growth rates, temperatures existed in the soil at which $M$. phragmitis would grow faster than M. bolleyi if additional factors such as competing fungi are not considered. In this context, temperature contributes to the differentiation of other Microdochium species $[47,48]$.

Other attributes may be involved in spatial niche differentiation for habitat type observed for Microdochium spp. Carbon usage patterns of the two species were found to overlap significantly more than expected by chance, although certain substrates, including compounds of the central carbon metabolism, secondary sugars, and sugar alcohols, are utilized differentially. In $P$. australis site-dependent variations for central metabolites were reported [49]. Basal culm internodes from flooded sites had higher total amino acid and lower total carbohydrate contents than those from dry sites. Several metabolites were individually recorded in that study, but none of those varying for habitat type could explain the contrasting habitat preferences of the two Microdochium species when considering the results of the BIOLOG experiments.

Earlier studies have noted that host-derived carbohydrates might affect the occurrences of plant-associated fungi. Root-associated endophytes grew better in vitro on low concentrations of certain carbohydrates than rhizosphere and soil fungi retrieved from the same Austrian grassland [50]. It was suggested, "that plant sugars or sugar alcohols may constitute signals that facilitate adaptation of certain fungi to a specific host plant". Some of such compounds are differentially utilizable by Microdochium spp. Another study reported that Neotyphodium endophytes were inhibited in vitro by high concentrations of hexose and were incapable of utilizing xylose and arabinose [51]. These findings were supported by results showing that Neotyphodium lolii 
grows more slowly in varieties of its host Lolium perenne bred for intrinsically high sugar concentrations [52]. For AM fungi, it was suggested that competition for the same carbon sources present in the same niche caused differential colonization [53]. A report comparing ericoid and orchid mycorrhizal fungi found that carbon source utilization was generally quite similar in vitro except for distinct differences for tannic acid and certain amino acids [54]. These publications indicate that the quality and the quantity of carbon sources available in the host may be one of the attributes influencing the composition of the associated fungal community.

Although the BIOLOG system provides interesting insights in the capacity of fungi to utilize various carbon sources, the difference in growth conditions in vitro compared to in planta should be considered. Single carbon sources are tested in vitro, whereas in planta many different sources are present. For the moment, it is not clear whether the carbon sources differentially used by Microdochium spp. in vitro are available at contrasting levels in roots or whether they have physiological importance for the fungi. Furthermore, competition with other endophytes for carbon sources may also influence their occurrences in the field. Thus, the challenging task remains to prove that differential utilization of carbon sources in vitro contributes to the coexistence of endophytes in planta.

Interactions between species implied by positive or negative co-occurrence was the third factor examined with respect to the differential colonization of the roots of common reed by Microdochium spp. Although spatial niche partitioning between $M$. bolleyi and $M$. phragmitis was significant, it was not perfect. Since none of the comparisons assessed by Fisher's Exact test exhibited any negative co-occurrence, a direct antagonism between these two species is unlikely. Moreover, in $8.4 \%$ of the samples both species were detected which may suggest "true" coexistence. Otherwise, reduced competition for space or carbon (or other essential compounds and ions) may explain this finding. This could occur if colony sizes were much smaller than sample sizes or if the two species used different resources. However, the two Microdochium species constitute only a small part of the entire fungal community colonizing common reed. Thus, antagonism or synergism might be indicated when considering additional fungi. This is already seen when including the data available for three more species. Whereas the EcoSim analysis suggests an overall signature of negative cooccurrence, Fisher's Exact test indicates negative and positive co-occurrences for certain species pairings. It is noteworthy that none of the three additional species exhibited negative co-occurrence with $M$. bolleyi and $M$. phragmitis in the total data set. Instead, $M$. bolleyi generally co-occurred significantly more frequently with Ms7Mb4 and Ms43Mb21 than expected by chance. Such a positive co-occurrence may appear when the conditions that are conducive for one species are also favorable for another species. Alternatively, positive co-occurrence may result from synergism. On the other hand, there existed an overall negative co-occurrence between Stagonospora sp. and Ms7Mb4, significantly preferring leaves [17] and roots [15], respectively. This could have resulted from strongly contrasting niche preferences, severe competition for the same substrates or from the secretion of toxins (antagonism). Our results suggest that it is rather unlikely that antagonism by any of the other three fungi is responsible for the differential colonization of roots by Microdochium spp. Since the fungal community on common reed is larger than addressed here, we cannot rule out that other endophytes may exert such influences.

\section{Conclusions}

This study supports the concept that niche partitioning allows for differential colonization of common reed by the fungal species investigated. Therefore, a purely neutral model is unlikely to explain the assembly of the mycoflora of common reed. Nonetheless, it remains to be shown to what extent stochastic factors could also contribute to variations in the composition, distribution and diversity of this fungal community.

\section{Additional material}

Additional file 1: Details of isolates studied. This file provides a list of 21 Microdochium isolates used in this study, including accession numbers of ITS sequences and information about their origins.

Additional file 2: Specificity of nested-PCR assays targeting Microdochium spp. This file documents the specificity of the assays employed. A) First PCR step using primers ITS1F and ITS4. M = $100 \mathrm{bp}$ size standard, water: no template DNA included, P. australis: genomic DNA of axenically grown reed plants, genomic DNAs from fungal isolates 4/97-9 (Humicola sp.), 6/97-38 (Chaetomium sp.), 6/97-54 (Fusarium sp.), A4 (Fusarium sp.), 5/97-16 (Microdochium phragmitis), 5/97-54 (M. bolleyi), 5/97-49 (M. bolleyi), 5/97-66 (M. phragmitis), respectively. B) and C) Second PCR steps using primers 5/97-16/TTS.F2 and 5/97-16/ITS.R2, and 5/97-54/ITS.F2 and 5/97-54/ITS.R2, respectively, and the products of the first PCR step as templates.

Additional file 3: Utilization of carbon sources. This file documents relative growth of Microdochium isolates on 95 different carbon sources on BIOLOG SF-N2 microtiter plates.

Additional file 4: Pair-wise analysis of spatial niche differentiation This file includes $P$-values from binomial distribution tests for pair-wise analysis of occurrence between five fungal species from reed with respect to space and time. This data set was used to create Figure 5A and $5 \mathrm{~B}$.

Additional file 5: Pair-wise analysis of co-occurrence. This file includes P-values from Fisher's Exact tests for pair-wise analysis of cooccurrence between five fungal species from reed with respect to space and time.

\section{Acknowledgements}

This work was financially supported by the Deutsche Forschungsgemeinschaft through SFB 454 (Bodenseelitoral). We thank Dr. 
Jan Nechwatal (Universität Konstanz) for providing the temperature data fo Lake Constance and for discussion of the data. We gratefully acknowledge Dr. Willi Nagl (Universität Konstanz) for advice on statistics, Dr. Ulrike Damm (CBS, Utrecht) for advice on taxonomy, and Michael Koch (Universität Konstanz) for technical help.

\section{Author details}

'Lehrstuhl Phytopathologie, Fachbereich Biologie, Universität Konstanz, Universitätsstr. 10, D-78457 Konstanz, Germany. ${ }^{2}$ Institut für Agrar- und Ernährungswissenschaften, Naturwissenschaftliche Fakultät III, Martin-LutherUniversität Halle-Wittenberg, Betty-Heimann-Str. 3, D-06120 Halle (Saale), Germany. ${ }^{3}$ Interdisziplinäres Zentrum für Nutzpflanzenforschung, MartinLuther-Universität Halle-Wittenberg, Betty-Heimann-Str. 3, D-06120 Halle (Saale), Germany.

\section{Authors' contributions}

ME collected samples, performed growth rate and nested-PCR assays, statistical data analyses, and contributed to the manuscript. KN collected samples, generated DNA sequences, and conducted the BIOLOG experiments. KWM was an advisor of the work and contributed to the manuscript. SGRW conceived and coordinated the project, contributed to statistical analyses, and wrote the manuscript. All authors read and approved the final manuscript.

Received: 11 June 2011 Accepted: 27 October 2011

Published: 27 October 2011

\section{References}

1. Hubbell SP: The unified neutral theory of biodiversity and biogeography. Princeton, NJ: Princeton University Press; 2001.

2. Bell G: The co-distribution of species in relation to the neutral theory of community ecology. Ecology 2005, 86:1757-1770.

3. Volkov I, Banavar JR, Hubbell SP, Maritan A: Neutral theory and relative species abundance in ecology. Nature 2003, 424:1035-1037.

4. Gilbert B, Lechowicz MJ: Neutrality, niches, and dispersal in a temperate forest understory. Proc Natl Acad Sci USA 2004, 101:7651-7656.

5. McGill BJ: A test of the unified neutral theory of biodiversity. Nature 2003, 422:881-885

6. Tilman D: Niche tradeoffs, neutrality, and community structure: a stochastic theory of resource competition, invasion, and community assembly. Proc Natl Acad Sci USA 2004, 101:10854-10861.

7. Cottenie K: Integrating environmental and spatial processes in ecological community dynamics. Ecol Lett 2005, 8:1175-1182.

8. Helgason T, Fitter AH: Natural selection and the evolutionary ecology of the arbuscular mycorrhizal fungi (Phylum Glomeromycota). J Exp Bot 2009, 60:2465-2480.

9. Parniske M: Arbuscular mycorrhiza: the mother of plant root endosymbioses. 2008, 6:763-775.

10. Rodriguez RJ, White JF Jr, Arnold AE, Redman RS: Fungal endophytes: diversity and functional roles. New Phytol 2009, 182:314-330.

11. Saikkonen K, Lehtonen P, Helander M, Koricheva J, Faeth SH: Model systems in ecology: dissecting the endophyte-grass literature. Trends Plant Sci 2006, 11:428-433.

12. Schardl $\mathrm{CL}$, Leuchtmann A, Spiering MJ: Symbioses of grasses with seedborne fungal endophytes. Annu Rev Plant Biol 2004, 55:315-340.

13. Schulz B, Boyle C: The endophytic continuum. Mycol Res 2005, 109:661-686.

14. Wirsel SGR: Homogenous stands of a wetland grass harbour diverse consortia of arbuscular mycorrhizal fungi. FEMS Microbiol Ecol 2004, 48:129-138.

15. Neubert $K$, Mendgen K, Brinkmann H, Wirsel SGR: Only a few fungal species dominate highly diverse mycofloras associated with the common reed. Appl Environ Microbiol 2006, 72:1118-1128.

16. Wirsel SGR, Leibinger W, Ernst M, Mendgen K: Genetic diversity of fungi closely associated with common reed. New Phytol 2001, 149:589-598.

17. Ernst M, Mendgen KW, Wirsel SGR: Endophytic fungal mutualists: Seedborne Stagonospora spp. enhance reed biomass production in axenic microcosms. Mol Plant-Microbe Interact 2003, 16:580-587.

18. Damm U, Brune A, Mendgen K: In vivo observation of conidial germination at the oxic-anoxic interface and infection of submerged reed roots by Microdochium bolleyi. FEMS Microbiol Ecol 2003, 45:293-299.
19. Hodges CF, Campbell DA: Infection of adventitious roots of Agrostis palustris by Idriella bolleyi. J Phytopathol 1996, 144:265-271.

20. Dawson WAJM, Bateman GL: Fungal communities on roots of wheat and barley and effects of seed treatments containing fluquinconazole applied to control take-all. Plant Pathol 2001, 50:75-82.

21. Fernandez MR, Holzgang G: Fungal populations in subcrown internodes and crowns of oat crops in Saskatchewan. Can J Plant Sci 2009, 89:549-557.

22. Wirsel SGR, Runge-Froböse C, Ahren DG, Kemen E, Oliver RP, Mendgen KW: Four or more species of Cladosporium sympatrically colonize Phragmites australis. Fungal Genet Biol 2002, 35:99-113.

23. Swofford DL: PAUP*. Phylogenetic Analysis Using Parsimony (* and Other Methods). Sunderland, MA: Sinauer; Version 42000.

24. Gotelli NJ, Entsminger GL: EcoSim: Null models software for ecology.

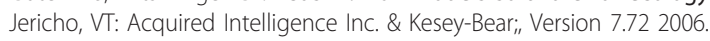

25. Ulrich W, Gotelli NJ: Null model analysis of species nestedness patterns. Ecology 2007, 88:1824-1831.

26. Rao PS, Niederpruem DJ: Carbohydrate metabolism during morphogenesis of Coprinus lagopus (sensu Buller). J Bacteriol 1969, 100:1222-1228.

27. Zervakis Gl, Moncalvo JM, Vilgalys R: Molecular phylogeny, biogeography and speciation of the mushroom species Pleurotus cystidiosus and allied taxa. Microbiology 2004, 150:715-726.

28. O'Brien HE, Parrent JL, Jackson JA, Moncalvo JM, Vilgalys R: Fungal community analysis by large-scale sequencing of environmental samples. Appl Environ Microbiol 2005, 71:5544-5550.

29. Smith ME, Douhan GW, Rizzo DM: Intra-specific and intra-sporocarp ITS variation of ectomycorrhizal fungi as assessed by rDNA sequencing of sporocarps and pooled ectomycorrhizal roots from a Quercus woodland. Mycorrhiza 2007, 18:15-22.

30. Park JW, Crowley DE: Nested PCR bias: a case study of Pseudomonas spp. in soil microcosms. J Environ Monit 2010, 12:985-988.

31. Fitt BDL, Huang YJ, van den Bosch F, West JS: Coexistence of related pathogen species on arable crops in space and time. Annu Rev Phytopathol 2006, 44:163-182.

32. Mummey DL, Rillig MC: Spatial characterization of arbuscular mycorrhizal fungal molecular diversity at the submetre scale in a temperate grassland. FEMS Microbiol Ecol 2008, 64:260-270.

33. Lekberg Y, Koide RT, Rohr JR, Aldrich-Wolfe L, Morton JB: Role of niche restrictions and dispersal in the composition of arbuscular mycorrhizal fungal communities. J Ecol 2007, 95:95-105.

34. Genney DR, Anderson IC, Alexander IJ: Fine-scale distribution of pine ectomycorrhizas and their extramatrical mycelium. New Phytol 2006, 170:381-390.

35. Dickie IA, Reich PB: Ectomycorrhizal fungal communities at forest edges. J Ecol 2005, 93:244-255.

36. Husband R, Herre EA, Turner SL, Gallery R, Young JPW: Molecular diversity of arbuscular mycorrhizal fungi and patterns of host association over time and space in a tropical forest. Mol Ecol 2002, 11:2669-2678.

37. Grunig CR, Sieber TN, Rogers SO, Holdenrieder O: Spatial distribution of dark septate endophytes in a confined forest plot. Mycol Res 2002, 106:832-840.

38. Queloz V, Grunig CR, Sieber TN, Holdenrieder O: Monitoring the spatial and temporal dynamics of a community of the tree-root endophyte Phialocephala fortinii s.l. New Phytol 2005, 168:651-660.

39. Carroll G: Forest Endophytes - Pattern and Process. Can J Bot 1995, 73 S1316-S1324.

40. Van Ryckegem G, Gessner MO, Verbeken A: Fungi on leaf blades of Phragmites australis in a brackish tidal marsh: Diversity, succession, and leaf decomposition. Microb Ecol 2007, 53:600-611.

41. Nechwatal J, Wielgoss A, Mendgen K: Diversity, host, and habitat specificity of oomycete communities in declining reed stands (Phragmites australis) of a large freshwater lake. Mycol Res 2008, 112:689-696.

42. Arnold AE, Mejia LC, Kyllo D, Rojas El, Maynard Z, Robbins N, Herre EA Fungal endophytes limit pathogen damage in a tropical tree. Proc Natl Acad Sci USA 2003, 100:15649-15654.

43. Osono T: Endophytic and epiphytic phyllosphere fungi of Camellia japonica: seasonal and leaf age-dependent variations. Mycologia 2008, 100:387-391. 
44. Schadt CW, Martin AP, Lipson DA, Schmidt SK: Seasonal dynamics of previously unknown fungal lineages in tundra soils. Science 2003, 301:1359-1361.

45. Nikolcheva LG, Bärlocher F: Seasonal and substrate preferences of fungi colonizing leaves in streams: traditional versus molecular evidence. Environ Microbiol 2005, 7:270-280.

46. Wielgoss A, Nechwatal J, Bogs C, Mendgen K: Host plant development, water level and water parameters shape Phragmites australis-associated oomycete communities and determine reed pathogen dynamics in a large lake. FEMS Microbiol Ecol 2009, 69:255-265.

47. Simpson DR, Thomsett MA, Nicholson P: Competitive interactions between Microdochium nivale var. majus, M. nivale var. nivale and Fusarium culmorum in planta and in vitro. Environ Microbiol 2004, 6:79-87.

48. Hofgaard IS, Wanner LA, Hageskal G, Henriksen B, Klemsdal SS, Tronsmo AM: Isolates of Microdochium nivale and M. majus differentiated by pathogenicity on perennial ryegrass (Lolium perenne L.) and in vitro growth at low temperature. J Phytopathol 2006, 154:267-274.

49. Koppitz $\mathrm{H}$ : Effects of flooding on the amino acid and carbohydrate patterns of Phragmites australis. Limnologica 2004, 34:37-47.

50. Hadacek F, Kraus GF: Plant root carbohydrates affect growth behaviour of endophytic microfungi. FEMS Microbiol Ecol 2002, 41:161-170.

51. Naffaa W, Ravel C, Guillaumin JJ: Nutritional requirements for growth of fungal endophytes of grasses. Can J Microbiol 1998, 44:231-237.

52. Rasmussen S, Parsons AJ, Bassett S, Christensen MJ, Hume DE, Johnson LJ, Johnson RD, Simpson WR, Stacke C, Voisey CR, et al: High nitrogen supply and carbohydrate content reduce fungal endophyte and alkaloid concentration in Lolium perenne. New Phytol 2007, 173:787-797.

53. Vandenkoornhuyse $P$, Mahe $S$, Ineson P, Staddon P, Ostle N, Cliquet JB, Francez AJ, Fitter AH, Young JPW: Active root-inhabiting microbes identified by rapid incorporation of plant-derived carbon into RNA. Proc Natl Acad Sci USA 2007, 104:16970-16975.

54. Midgley DJ, Jordan LA, Saleeba JA, McGee PA: Utilisation of carbon substrates by orchid and ericoid mycorrhizal fungi from Australian dry sclerophyll forests. Mycorrhiza 2006, 16:175-182.

doi:10.1186/1471-2180-11-242

Cite this article as: Ernst et al:: Niche differentiation of two sympatric species of Microdochium colonizing the roots of common reed. BMC Microbiology 2011 11:242.

\section{Submit your next manuscript to BioMed Central and take full advantage of:}

- Convenient online submission

- Thorough peer review

- No space constraints or color figure charges

- Immediate publication on acceptance

- Inclusion in PubMed, CAS, Scopus and Google Scholar

- Research which is freely available for redistribution

Submit your manuscript at www.biomedcentral.com/submit 